\title{
Can childcare work be designed to promote moderate and vigorous physical activity, cardiorespiratory fitness and health? Study protocol for the Goldilocks- childcare randomised controlled trial
}

Mark Lidegaard ${ }^{1,2^{*}}$ (D, Anders Fritz Lerche ${ }^{1}$, Pernille Kold Munch', Kathrine Greby Schmidt', Charlotte Lund Rasmussen ${ }^{1,3}$, Charlotte Diana Nørregaard Rasmussen ${ }^{1}$, Svend Erik Mathiassen ${ }^{4}$, Leon Straker ${ }^{5}$ and Andreas Holtermann ${ }^{1,6}$

\begin{abstract}
Background: Despite extensive efforts, issues like obesity and poor physical capacity remain challenges for a healthy work life in several occupations. The Goldilocks work principle offers a new approach, encouraging design of productive work to promote physical capacity and health. This paper presents the protocol for the Goldilockschildcare study, a randomised controlled intervention trial aiming to evaluate the effectiveness of implementing the Goldilocks work principle in childcare. The primary aim of the intervention is to increase time in moderate to vigorous physical activity (MVPA) by having the childcare workers act as active role models for children in daily playful physical activities, and thereby improve cardiorespiratory fitness and health of the workers.

Methods: The study is a cluster-randomised trial with a usual-practice wait-list control group. The 10-week intervention consists of two phases. In the first, the childcare workers will participate in two participatory workshops aiming to a) develop playful physical activities ('Goldilocks-games') for children in which childcare workers participate as active role models at MVPA intensity, and b) develop action plans for implementation of the Goldilocks-games in daily work routines. In the second phase, childcare institutions will implement the Goldilocks-games. The primary outcome is working time spent in MVPA, and secondary outcomes are cardiorespiratory fitness, sleeping heart rate, perceived need for recovery, and productivity. Primary outcome and process evaluation will be based on direct measurements of physical activity and heart rate, determination of cardiorespiratory fitness, and questionnaires.
\end{abstract}

Discussion: If proven effective, the Goldilocks work principle has a large potential for promoting sustainable health and working lives of childcare workers.

Trial registration: ISRCTN, ISRCTN15644757, Registered 25th December 2019

Keywords: Ergonomics, Workplace intervention, Cardiometabolic fitness, Physical activity, Physical work demand, Productive work, Sedentary behaviour, Workplace health promotion

\footnotetext{
* Correspondence: vmlg@novonordisk.com

${ }^{1}$ National Research Centre for the Working Environment, Copenhagen,

Denmark

${ }^{2}$ Novo Nordisk A/S, Novo Nordisk Health \& Safety, Bagsværd, Denmark

Full list of author information is available at the end of the article
}

(c) The Author(s). 2020 Open Access This article is distributed under the terms of the Creative Commons Attribution 4.0 International License (http://creativecommons.org/licenses/by/4.0/), which permits unrestricted use, distribution, and reproduction in any medium, provided you give appropriate credit to the original author(s) and the source, provide a link to the Creative Commons license, and indicate if changes were made. The Creative Commons Public Domain Dedication waiver (http://creativecommons.org/publicdomain/zero/1.0/) applies to the data made available in this article, unless otherwise stated. 


\section{Background}

Despite extensive efforts to promote occupational health, several occupations still face considerable challenges in achieving a long, healthy, and sustainable work life for those employed. Examples of these challenges are the increased occurrence of obesity in the working population [1], and a considerable proportion of workers not having the physical capacity required for performing their main work tasks [2]. Both issues are associated with social inequality in health [3], and particularly pronounced in an ageing working population [4].

The dominant approach to prevent work-related disorders has consisted of reducing the physical activity demands at work [5]. Accordingly, the intensity of occupational physical activity has been minimised in many occupations, most often by increasing the amount of sedentary time [6]. To compensate for insufficient physical activity at work, numerous workplace health promotion initiatives have then attempted to improve employees' physical capacity and health by offering physical exercise at the workplace $[7,8]$. However, these initiatives have not been successful in reaching the employees most in need [9-11]. Also, workplace physical exercise programmes are often not appealing to employers, since they require time away from productive work, and thus being a costly initiative. Therefore, both minimising physical activity and introducing exercising during working hours are inadequate to solve the abovementioned challenges in occupational health [12].

As an alternative, the Goldilocks work principle has been proposed $[12,13]$, aiming to design productive work in a way that promotes physical capacity and health without compromising productivity [12, 13]. Building on work physiology fundamentals [14], the Goldilocks work principle seeks to achieve a 'just right' balance between physical activity demands and recovery at work, so that a training effect can be obtained from the work per se, leading to better health [12]. However, the effectiveness of the Goldilocks work principle remains to be evaluated in randomised controlled trials.

Childcare workers generally report a high prevalence of poor general health, physical work exertion, musculoskeletal pain, and sickness absence [15-17]. Moreover, childcare workers have been shown to spend only a small amount of work time in moderate to vigorous physical activity (MVPA) [18]. In a pilot study conducted among childcare workers, we found workers were sitting extensively and had minimal work time in MVPA. Because MVPA is well documented to improve cardiorespiratory fitness and health [19], we believe that an increase in MVPA in the daily routines of childcare workers could have a great potential to lead to better health and more sustainable work.
An important general aim in childcare is to encourage children to be more physically active $[20,21]$. Therefore, by acting as active role models in daily playful physical activities (termed 'Goldilocks-games') together with the children, the childcare workers may be able to achieve sufficient daily work time in MVPA to improve their own cardiorespiratory fitness and health. However, this has not been evaluated in a randomised controlled trial among childcare workers.

The aim of this protocol paper is to describe the design, implementation, and evaluation of the participatory randomised controlled trial 'Goldilocks-childcare', which seeks to increase the childcare workers' productive work time spent in MVPA, and thus their cardiorespiratory fitness and health.

\section{Methods/ design}

Data protection, ethical approval, and trial registration The National Research Centre for the Working Environment has an institutional agreement with the Danish Data Protection Agency about procedures to treat confidential data (journal number 2015-41-4232), e.g. by securing data at a protected drive with limited access, and by anonymizing all individual data.

The Danish National Committee on Biomedical Research Ethics (The local ethical committee of Frederiksberg and Copenhagen) has evaluated a description of the study and concluded that, according to Danish law as defined in Committee Act $\mathbb{} 2$ and $\mathbb{1} 1$, the intervention described should not be further reported to the local ethics committee (Ref number: H-18041423).

The study is registered in the International Standard Registered Clinical/soCial sTudy Number (ISRCTN) registry (ISRCTN15644757). The protocol conforms to the Standard Protocol Items: Recommendations for Interventional Trials (SPIRIT) 2013 statement [22] and the Template for Intervention Description and Replication (TIDieR) checklist [23]. The reporting of the study will follow the Consolidated Standards of Reporting Trials (CONSORT) 2010 statement [24].

\section{Study design}

Figure 1 provides an overview of the study design. The study will use a cluster-randomised design with a usualpractice wait-list control group. As the study is an organisational intervention in a work place setting, individual randomisation is neither possible nor appropriate [25]. Thus, each participating institution will form a cluster. A wait-list design (offering the intervention to the intervention and, eventually, even to the control groups) was chosen in an attempt to minimise any potential lack of commitment from an institution acting as control [26]. Thus, we will randomly assign the participating childcare institutions to, i) the intervention group 


\section{Intervention group}

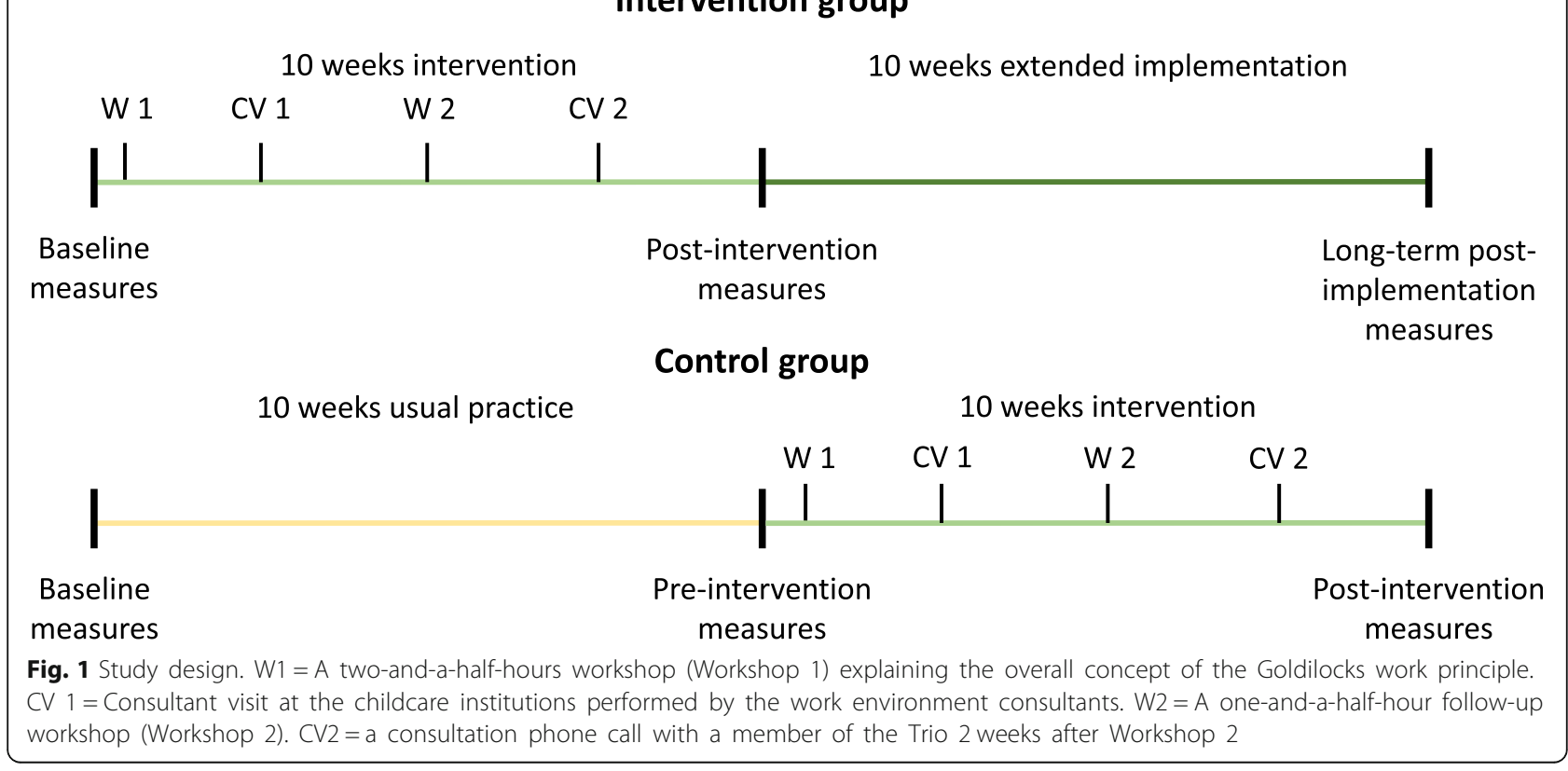

immediately receiving the intervention after the baseline measurements or, ii) the control group continuing usual practice for 10 weeks after the baseline measurements and then performing the intervention. Each intervention period will last 10 weeks. After the 10 weeks intervention for the intervention group, they will be encouraged to continue with Goldilocks-games without further support from the research team.

Recruitment of childcare institutions opened in March 2019 and will continue until the sufficient number of childcare institutions required for the trial are enrolled. Recruitment of participants at the participating childcare institutions will open in January 2020. The intervention will start in January 2020 with the participants enrolled in the childcare institutions recruited first. The last childcare institutions enrolled in the trial will start the intervention by September 2020.

\section{Study population}

The childcare institutions are recruited from the greater Copenhagen area in collaboration with employer organisations, unions, and local government municipalities. In order to be eligible for participation, the childcare institution should employ a minimum of nine childcare workers.

As the intervention is organisational, all employees within the participating childcare institutions will take part in the intervention activities, and all childcare workers will be eligible for participation in the trial evaluation. Since this participation is voluntary, the childcare workers will, prior to entering the trial, be provided with information about the trial, asked if they agree to participate, and if so, asked to sign an informed consent form.

\section{Randomisation and blinding}

Cluster-randomisation will be used with each childcare institution constituting a cluster, in order to avoid contamination within an institution between participating and non-participating workers. Participating childcare institutions will be randomised to either intervention or usual practice (i.e. wait-list control) arms of the trial. This randomisation will be conducted upon enrolment of each participating childcare institution into the study. The randomisation sequence has been developed using the statistical software R [27].

Because of the time needed for the childcare institutions to plan their participation in the trial, we need to inform them about their allocation to intervention or control group before the baseline data collection. Moreover, due to the nature of the trial, it will not be possible to blind neither the researchers nor the participating employees as regards whether a particular institution is subject to the intervention or not. However, allocation concealment will be maintained throughout the study, and all researchers conducting the randomisation, statistical analysis, and evaluation will be blinded.

\section{Intervention}

The overall study idea was developed in collaboration between the researchers and work environment consultants (Physiotherapists and Occupational therapists) from the Work Environment Consultancy of Copenhagen 
Municipality (WECoCM), based on the Goldilocks work principle [12].

To ensure that the intervention is relevant for, tailored to, and closely integrated with pedagogical teaching aims as well as feasible for the childcare institutions, the intervention will apply a participatory approach. The Goldilocks work principle for the intervention content and implementation is explained and modified on the basis of a dialog with stakeholders related to childcare (e.g. employer organisations and unions, practitioners in childcare, work environment consultants), of observations of childcare work, and of a dialog with managers and employees in childcare institutions.

Further, the manager, a union representative, and an occupational health and safety representative (collectively referred to as the Trio) from each of the participating childcare institutions will attend a workshop. The workshop will outline the Goldilocks work principle for all institutions and aim to facilitate planning and management support to the implementation and evaluation of the intervention. The Trio will then be involved in planning and tailoring the intervention process to their own childcare institution. Thus, the Trio is responsible for outlining the pedagogical focus, rostering and practical planning at their institutions. Involving the Trio at an early stage will enhance the likelihood of organisational buy-in and the possibility for introducing organisational changes.

To facilitate development and implementation of the Goldilocks-games, we will conduct a proof of concept study in a few childcare institutions. The proof of concept study will focus on development and evaluation of feasible Goldilocks-games that can increase the occurrence of MVPA among the childcare workers. Experiences from this proof of concept study will be applied in the first workshop at each institution participating in the randomised controlled trial.

A programme logic model for the Goldilocks work principle was developed (Fig. 2). The programme logic model provides a schematic overview of the steps from introducing the Goldilocks work intervention to the effects on cardiorespiratory fitness and health of the childcare workers. In addition, the programme model assists in guiding the effect and process evaluation.

\section{Delivery of intervention}

Work environment consultants (Physiotherapists and Occupational therapists) from the Work Environment Consultancy of Copenhagen Municipality (WECoCM) will deliver the intervention components (workshops and visits). In order to ensure a consistent delivery across the participating institutions, we have developed an intervention protocol describing all intervention components.

\section{Intervention content}

Figure 3 provides an overview of the intervention components. At each individual childcare institution, the intervention will be initiated by a two-and-a-half-hours workshop (Workshop 1) during a regular staff meeting.

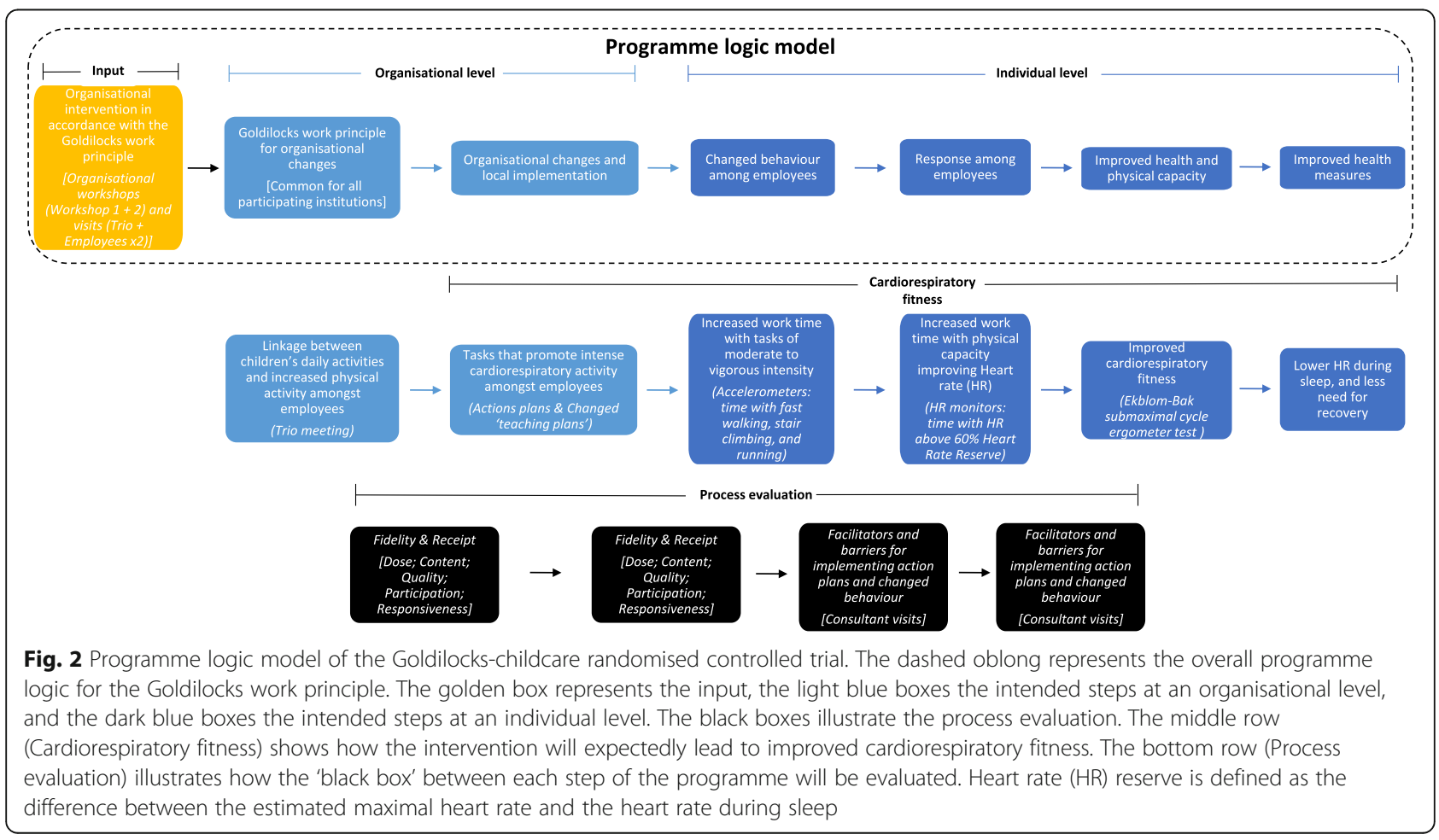




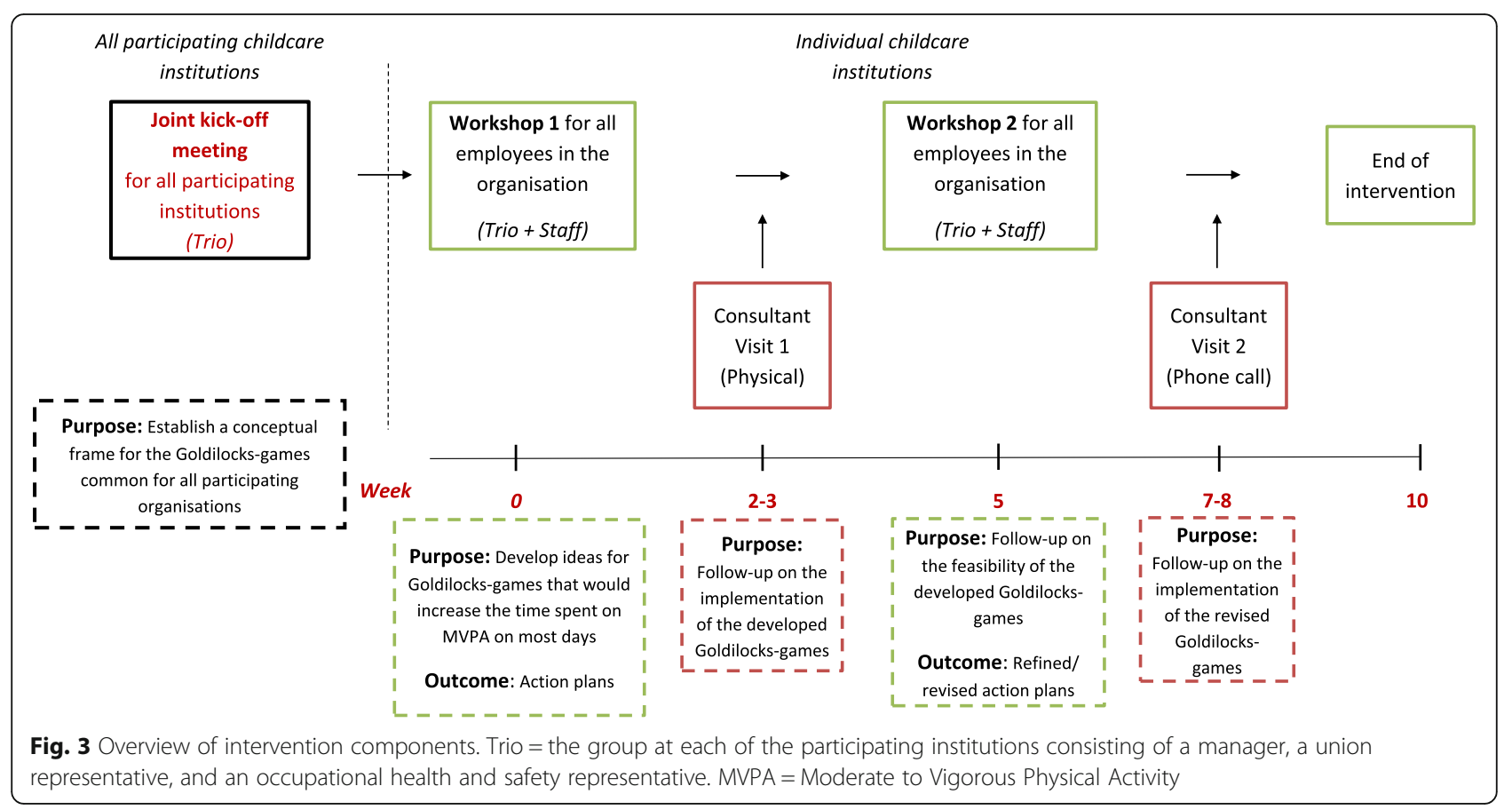

At Workshop 1, the work environment consultants will inform the participating Trio and childcare workers about the overall concept of the Goldilocks work principle, facilitate that the participants develop tailored Goldilocks-games compliant with their pedagogical teaching goals, and finally develop specific action plans allocating responsibilities for implementation of the Goldilocks-games in their daily routines and schedules. Information regarding whether the Goldilocks-games were conducted as planned will be collected.

After three to four weeks of the intervention period, the work environment consultants will conduct a oneand-a-half-hour follow-up workshop (Workshop 2) with the participants at each individual childcare institution. The aims will be to i) evaluate the implementation of the Goldilocks-games, ii) facilitate sustainability of wellfunctioning Goldilocks-games, and iii) modify those Goldilocks-games, which are not working as intended. Moreover, in order to facilitate implementation of the Goldilocks-games, the work environment consultants will make a consultation visit at the childcare institution 2 weeks after Workshop 1 and have a consultation phone call with a member of the Trio 2 weeks after Workshop 2.

\section{Control group}

Institutions in this group serve as waitlist controls. Thus, institutions in the control group will continue their usual practice for the first 10 weeks, while institutions in the intervention group implement the intervention. Following the 10-week period, control group institutions will implement the intervention in the same manner as those in the intervention group.

\section{Data collection}

Data will be collected at three time points: At baseline; at a 10-week follow-up (Immediate post-intervention for intervention group and Pre-intervention for control group); and at a 20-week follow-up (Long-term postintervention for intervention groups and Immediate post-intervention for control group). Baseline and immediate post-intervention data collection will consist of i) an electronic questionnaire through a link provided in a text message to the participants, ii) anthropometric measurements, iii) testing of cardiorespiratory fitness, and iv) wearable sensor technical measurements of physical activity and heart rate. At the long-term post-intervention follow-up, data collection will include only a modified questionnaire.

\section{Questionnaire}

The questionnaires include a combination of items to assess the following descriptive factors of the study population, as well as potential confounders, and beneficial and adverse effects i) sociodemographic factors, i.e. age; sex; ethnicity; length of service; job title; and weekly working hours, ii) health and behaviours, i.e. musculoskeletal pain and pain-related work interference [28]; medicine use; smoking; general health [29, 30]; selfefficacy [31]; well-being [32]; and sleep behaviour [33], iii) self-rated physical capacity: cardiorespiratory fitness and muscle strength [34], iv) stress [35], and v) work 
environment factors, i.e. perceived physical exertion during work [36]; productivity [37]; psychosocial work environment [38]; single item work ability [39]; shortversion perceived need for recovery [40]; and sickness absence and presenteeism [41, 42].

Need for recovery and productivity will act as secondary outcomes. Need for recovery will be determined using a short, three-item version: 'At the end of my work day I am exhausted'; 'I find it hard to show interest in other people, when I have just come home from work'; and 'It takes me over an hour before I am fully recovered after a work day'. All items have five response categories: 'Never'; 'Rarely'; 'Some of the Time'; 'Most of the Time'; and 'Always' [40]. Productivity will be determined using one item: 'On a scale from 0 to 10 , where 0 is the worst job performance anyone could have at your job and 10 is the performance of a top worker, how would you rate your overall job performance on the days you worked during the past 4 weeks (28 days)?' [47].

\section{Anthropometric measures}

We will measure height (Seca 213; Seca GmbH, Hamburg, Germany) and weight (BC-418 MA body composition analyzer; Tanita, Tokyo, Japan), and calculate the body mass index (body weight $[\mathrm{kg}] /$ (body height squared $\left.\left.\left[\mathrm{m}^{2}\right]\right)\right)$. In addition, we will determine fat percentage (BC418 MA body composition analyzer; Tanita, Tokyo, Japan) and resting blood pressure (Omron M3 or Omron M6 Comfort; Omron Corporation, Kyoto, Japan).

\section{Physical activity and heart rate \\ Physical activity type and body postures}

Physical activity type (i.e. moving, walking, running, bicycling, climbing stairs), body position (i.e. sitting, and standing), and number of steps will be measured using a thigh-worn AX3 accelerometer (3-Axis Logging Accelerometer; Axivity Ltd., Newcastle upon Tyne, UK) and processed using the validated Acti4 software [43-45].

The AX3 accelerometer generates measurements of linear acceleration in three dimensions with a dynamic range of $\pm 8 \mathrm{G}$, sampled with a precision of 13 bits at a sampling rate of $25 \mathrm{~Hz}$. The AX3 accelerometers are initialised prior to recording, and data will be downloaded using the manufacturer's software (OMGUI Version 1.0.0.30; Axivity Ltd).

One AX3 accelerometer will be mounted on the right thigh at the most muscular part of the quadriceps femoris, midway on the line between the anterior inferior iliac spine and the top of the patella [44]. The AX3 accelerometer will be mounted on the skin with adhesive tape (Hair-Set double-sided adhesive tape; $3 \mathrm{M}$ Company, Maplewood, MN, USA) and secured with transparent adhesive film (Opsite Flexifix; Smith \& Nephew plc, London, UK). We will ask the participants to wear the accelerometer around the clock during five working days.

\section{Heart rate and heart rate variability measures}

We will measure heart rate and heart rate variability using a Firstbeat Bodyguard 2 monitor (Firstbeat Technologies Ltd., Jyväskylä, Finland). The monitor measures the electrocardiogram at a sampling frequency of 1000 $\mathrm{Hz}$, and the signal is processed to identify R-spikes and subsequently $R-R$ intervals. The monitor has been validated for long-term measurements of heart rate in free living [46].

The Firstbeat Bodyguard 2 will be mounted with $\mathrm{Ag} /$ $\mathrm{AgCl}$ pre-gelled electrodes (Ambu WhiteSensor CMM00-S/30; Ambu A/S, Ballerup, Denmark) below the right clavicle and at the left rib cage. We will download data from Firstbeat Bodyguard 2 using the manufacturer's software (Firstbeat Uploader Version 3.1.2.0; Firstbeat Technologies Ltd., Jyväskylä, Finland). As for the accelerometer, we will ask the participants to wear the heart rate sensor around the clock during five working days.

\section{Cardiorespiratory fitness}

Cardiorespiratory fitness will be assessed using the Ekblom-Bak submaximal test [47] performed on a cycle ergometer (Monark AB, Varberg, Sweden). The Ekblom-Bak test estimates cardiorespiratory fitness $\left(\mathrm{VO}_{2 \max }\right)$ based on the difference in heart rate between an initial low standard workload and a subsequent higher 'final' workload. The test has shown good validity in a wide population range (Women: 21-86 years old with a $\mathrm{VO}_{2 \max }$ range of $19-62 \mathrm{ml} /$ $\mathrm{min} / \mathrm{kg}$; Men: 20-84 years old with a $\mathrm{VO}_{2 \max }$ range of $24-76 \mathrm{ml} / \mathrm{min} / \mathrm{kg}$ [47]).

The test is initiated by having participants performing a standard workload of 60 revolutions/ minute at a resistance of $0.5 \mathrm{kp}$ for $4 \mathrm{~min}$. Heart rate is measured four times during the last minute $(3: 15,3: 30,3: 45$, and $4: 00)$ and the average of these four measurements is used as the initial heart rate. Subsequently, resistance is increased in steps with the aim of reaching a perceived exertion rating of approximately 14 on the Borg RPE-scale [36], and a heart rate between 120 and 150 , or 110 and 140 beats/ minute for participants younger or older than 50 years, respectively. Perceived exertion is rated after 2 min at each step. If below 10 or 12 , resistance is incrementally increased by $1 \mathrm{kp}$ or $0.5 \mathrm{kp}$, respectively, for younger and older participants. When reaching the target exertion and heart rate, four measurements are collected during the last minute, with the average heart rate of these four measurements providing the final heart rate [47]. Cardiorespiratory fitness is calculated from equations described in [47]. 


\section{Primary and secondary outcomes}

Differences between the intervention and the control groups in changes from baseline to 10-week follow-up will be evaluated for all primary and secondary outcomes. The primary outcome is the relative work time spent in MVPA, as determined by either heart rate (i.e. $\geq 60 \%$ of heart rate reserve) or accelerometer recordings (i.e. fast walking ( $\geq 130$ steps/ minute), running, or stair climbing).

Furthermore, the study has four secondary outcomes: i) cardiorespiratory fitness, ii) resting heart rate during sleep, iii) perceived need for recovery; and iv) selfreported productivity. Figure 4 shows the SPIRIT schedule of enrolment, interventions, and assessments [22].

\section{Economic evaluation}

The economic evaluation aims to determine the costeffectiveness of the intervention in terms of cost (from the employer's perspective) per increment in work time spent in MVPA. Costs of the intervention will include costs related to implementation and operation, specifically:

\section{Staff time}

Participation in intervention activities for workers (workshops, and kick-off meeting for the project), and Trio (workshops, kick-off meeting for the project, and time spent on planning the logistics of implementing the intervention) will be assessed based on registration of attendance. Costs for participants and supervisors will subsequently be estimated based on their average yearly gross salaries, including overhead.

\section{Consultant time}

Time spent on delivery of the intervention (workshops, kick-off meeting, and workplace visits) will be assessed by asking the work environment consultants how many hours they spent on these activities, including preparations. The number of working hours will be valued by using their hourly fee, including overhead.

\section{Consumables}

Materials, such as printouts and posters, as well as fruit/ snacks/coffee consumed at meetings will be noted. Costs will be valued using invoices.

For the control group, only costs related to participation in the joint kick-off meeting will be included.

The total intervention costs for the employer will be estimated and compared between the intervention and control group. The incremental cost-effectiveness ratio will be calculated by dividing the mean difference in costs (incremental cost) between both groups by the difference in effects (incremental effect) on the primary outcome measure.
In addition, sickness absence will be measured by questionnaire from the participants in the control and intervention groups at baseline and 10-week follow-up.

Costs associated with absenteeism will be estimated using the Friction-Cost approach [48]. A friction period of 4 weeks will be assumed, as the Danish social security system takes over costs after 4 weeks of sickness absence. Furthermore, an appropriate elasticity factor will be used. Health-related productivity losses will be valued using gross yearly salaries of the participants converted to a daily cost based on assumed numbers of working days per year.

\section{Process evaluation}

Before the intervention starts, we will collect information addressing facilities at the childcare institutions, including possible areas for the Goldilocks-games and accessibility to these areas. Further, we will collect information about readiness for change among employees at the childcare institutions.

To assess the extent to which the intervention is implemented as intended, a process evaluation will be conducted. The process evaluation will follow the principles described by Steckler and Linnan (2002), and Ferm and colleagues (2018) [49, 50]. The process evaluation will assess how the intervention was delivered (Fidelity) and received (Receipt) [49].

Fidelity includes three measures i) Dose (the number of intervention components delivered); ii) Content (if the components are delivered in accordance with the workshop manual); and iii) Quality (the self-rated performance of the deliverer). Receipt includes two measures i) Participation (number of participants attending the two workshops); and ii) Responsiveness (satisfaction and motivation among the participants). Following each workshop, the work environment consultants will assess dose, content, quality, and participation in a customised questionnaire. Responsiveness will be assessed by questionnaire to the participants following the intervention.

Additionally, we will collect information regarding facilitators and barriers for implementing the intervention through semi-structured interviews with managers during the consultant visits. These interviews will provide information on contextual factors for each of the childcare institutions that could have influenced the implementation or the effect of the intervention, e.g. occurrence of major organisational changes during the intervention period, or concurrent activities with a likely impact.

\section{Power calculation}

We estimated the number of participants to be included in the trial based on a statistical power analysis of the primary outcome using clustered parallel groups with before-and-after measures to determine the design 


\begin{tabular}{|c|c|c|c|c|c|c|c|c|}
\hline \multirow[b]{3}{*}{ TIMEPOINT } & \multicolumn{8}{|c|}{ STUDY PERIOD } \\
\hline & \multirow[t]{2}{*}{ Allocation } & \multirow[t]{2}{*}{ Enrolment } & \multicolumn{5}{|c|}{ Post-allocation } & \multirow{2}{*}{\begin{tabular}{|l|} 
Close-out \\
$20 \mathrm{FU}$ \\
\end{tabular}} \\
\hline & & & $W 1$ & CV 1 & $W 2$ & $C V 2$ & $10 \mathrm{FU}$ & \\
\hline \multicolumn{9}{|l|}{ ENROLMENT: } \\
\hline \multirow{3}{*}{$\begin{array}{l}\text { Eligibility screen } \\
\text { Informed consent } \\
\text { Randomisation* }\end{array}$} & & $\mathrm{X}$ & & & & & & \\
\hline & & $\mathrm{X}$ & & & & & & \\
\hline & $\mathrm{X}$ & & & & & & & \\
\hline Allocation & $\mathrm{x}$ & & & & & & & \\
\hline \multicolumn{9}{|l|}{ INTERVENTIONS: } \\
\hline \multirow{2}{*}{$\begin{array}{l}\text { Intervention } \\
\text { Control** }^{*}\end{array}$} & & & $\leftarrow$ & & & & $\longrightarrow$ & \\
\hline & & & $\curvearrowleft$ & & & & $\rightarrow$ & \\
\hline \multicolumn{9}{|l|}{ ASSESSMENTS: } \\
\hline \multirow{3}{*}{$\begin{array}{l}\text { Demographics } \\
\text { Anthropometric measures } \\
\text { Primary outcomes }\end{array}$} & & $\mathrm{X}$ & & & & & & \\
\hline & & $\mathrm{X}$ & & & & & $\mathrm{X}$ & $\mathrm{X}$ \\
\hline & & & & & & & & \\
\hline $\begin{array}{l}\text { Time with MVPA during } \\
\text { working hours }\end{array}$ & & $\mathrm{X}$ & & & & & $\mathrm{X}$ & $\mathrm{X}$ \\
\hline \multicolumn{9}{|l|}{ Secondary outcomes } \\
\hline \multirow{2}{*}{$\begin{array}{l}\text { Cardiorespiratory fitness } \\
\text { Heart rate during sleep }\end{array}$} & & $\mathrm{X}$ & & & & & $\mathrm{X}$ & $\mathrm{X}$ \\
\hline & & $\mathrm{X}$ & & & & & $\mathrm{X}$ & $\mathrm{X}$ \\
\hline Need for recovery & & $\mathrm{X}$ & & & & & $\mathrm{X}$ & $\mathrm{X}$ \\
\hline \multirow{2}{*}{$\begin{array}{l}\text { Self-perceived productivity } \\
\text { Economic measures }\end{array}$} & & $\mathrm{X}$ & & & & & $\mathrm{X}$ & $\mathrm{X}$ \\
\hline & & & & & & & & \\
\hline Absenteeism & & $\mathrm{X}$ & & & & & & $\mathrm{X}$ \\
\hline Presenteeism & & $\mathrm{X}$ & & & & & & \\
\hline Costs & & & & & & & & $\mathrm{X}$ \\
\hline \multicolumn{9}{|l|}{ Process evaluation } \\
\hline Fidelity & & & $\mathrm{X}$ & & $\mathrm{X}$ & & & \\
\hline Receipt & & & $\mathrm{X}$ & & $\mathrm{X}$ & & & \\
\hline Contextual factors & & & & $\mathrm{X}$ & & $\mathrm{X}$ & & \\
\hline
\end{tabular}

Fig. 4 Standard Protocol Items: Recommendation for Interventional Trials (SPIRIT) schedule of enrolment, interventions, and assessments. $\mathrm{W}=$ Workshop; $\mathrm{CV}=$ Consultant visit; FU = Follow up; MVPA = Moderate to Vigorous Physical Activity. *Randomisation carried out at childcare institution level before baseline measurements. ${ }^{* *}$ The study uses a wait-list design; control group institutions will receive the intervention after the intervention group

effect. The power calculation was based on data from a larger sample $(N=167)$ of childcare workers in Copenhagen in a previous trial (ISRCTN10928313) [51]. In this sample, work time spent with a heart rate reserve (HRR) $\geq 60 \%$ was, on average, $1.24 \mathrm{~min} /$ day with a standard deviation (SD) between subjects of 2.90. The power calculation was done after processing data according to the principles of compositional data analysis (CoDA) $[52,53]$, where work time spent at $\mathrm{HRR} \geq 60 \%$ is expressed relative to time spent at HRR $<60 \%$ using isometric log-ratios (ilr) [54-56]. Expressed as an ilr, the average relative work time spent at $H R R \geq 60 \%$ was $4.35(\mathrm{SD}=1.10)$.

Based on these transformed data, we will need an estimated total of 132 participants (corresponding to approximately 14 childcare institution clusters shared between the intervention and wait-list groups) to be able to detect (at $p<0.05$ ) a $5 \mathrm{~min} /$ day increase in relative work time spent at HRR $\geq 60 \%$ with a power of 0.80 , an estimated intra-cluster correlation coefficient (ICC) of 0.05 , a fixed cluster size of 10 , and an assumed drop-out rate of $30 \%$. 


\section{Statistical analysis}

Evaluation of intervention effectiveness on the primary and secondary outcomes as well as the cost-effectiveness will be based on multilevel models, taking into account that the study design implies repeated measurements within each participant [57]. Conclusions about the effectiveness of the intervention with respect to the primary and secondary outcomes will be based on the group effect and its 95\% confidence interval. The 95\% confidence intervals for the incremental cost-effectiveness ratio will be estimated using bootstrapping (1000 bootstrap samples with replacement) [58]. All analyses will be performed according to the intention-to-treat principle [59]. Potential confounding factors (e.g. baseline differences between the intervention and control group in factors like age and BMI) will be adjusted for in the statistical analyses.

\section{Discussion}

This will be the first randomised controlled trial to evaluate the effectiveness of the Goldilocks work principle in terms of increased work time in MVPA and better cardiorespiratory fitness for workers. If successful, the study will demonstrate that the Goldilocks work principle has a potential to improve health and physical capacity among workers while performing productive work. A scaling of the Goldilocks work principle to other occupations and countries could have a large impact on general health and social justice of working populations [12].

\section{Strengths and limitations of the study}

A methodological strength of the study is the use of a cluster randomised design, which minimises the risk of contamination within and between institutions in the intervention and control groups. Another methodological strength is the application of a systematic participatory approach involving the end users throughout the entire process. We will collect experiences and information during the process of developing and tailoring the intervention, and this can likely benefit the present as well as future studies. Even the use of wearable accelerometers and heart rate monitors for measuring the primary outcomes is a strength. By using wearable sensors rather than self-reported methods to record the primary outcome, bias introduced by self-reports, e.g. due to lack of blinding of participants or inaccurate perception [60], is considered minimal.

In addition, utilizing CoDA is a strength of this study, since it allows the co-dependency between work time spent in different behaviour types (sedentary behaviour; light physical activity; MVPA) to be taken into account. Thus, the use of CoDA represents an approach that gives a better understanding of the potential effect of an intervention from a whole-workday perspective than if each behaviour is analysed as an independent factor [56, 61]. Furthermore, conducting a process evaluation is a strength of this study. The process evaluation provides an opportunity for thoroughly evaluating the implementation of the intervention and identify why the intervention may or may not be effective.

The main limitation of the study is the lack of allocation concealment, which introduces the risk of selection bias. This is, however, inevitable since the study design requires the participating childcare institutions to make organisational changes that build on a participatory approach. Thus, the participating childcare institutions need to be informed of their allocation in advance, in order to facilitate their introduction of organisational changes as well as allowing them adequate time for preparing and initiating any required logistics, e.g. staffing issues or equipment. Also, blinding the participants is, for natural reasons, not possible. This is a common issue in participatory interventions, and it may introduce a risk of unintended effects, e.g. a possible placebo change in the selected outcome(s), or a Hawthorne effect [62]. However, we will try to minimise both of these limitations. All participants will, eventually, receive the intervention, participants are only informed about when the intervention will be implemented at their institution, and not whether they are allocated to an intervention or a control group in the actual intervention project. Thus, the lack of blinding is expected to have no or minimal influence on the results of the study.

\section{Trial status}

The study is ongoing. Recruitment of childcare institutions opened in March 2019 and will continue until all childcare institutions required for the trial are enrolled, planned to be September 2020. The intervention will start at the first enrolled childcare institutions in January 2020. For the last childcare institutions enrolled in the trial, the intervention is planned to start September 2020. We will finalize the intervention for the last group of childcare institutions in December 2020.

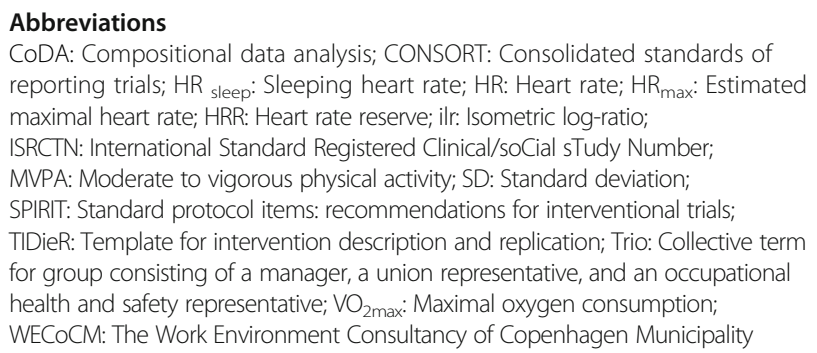

Acknowledgements

We acknowledge engineer Jørgen Skotte, physiologist Sandra Schade Jacobsen, and work environment consultants Vibeke Andersen, Cornelia Strøh, and Maja Schønheyder of the Work Environment Consultancy of 
Copenhagen Municipality for their valuable contribution to the development and planning of the study.

\section{Authors' contributions}

$\mathrm{ML}$ contributed to the conception and design of the study, wrote the initial protocol, and drafted the manuscript. AFL participated in discussions of the conceptual design of the study and drafting of the manuscript. PKM participated in discussions of the conceptual design of the study and drafting of the manuscript. KGS participated in discussions of the conceptual design of the study and drafting of the manuscript. CLR contributed to the conception and design of the study, wrote the funding application and performed the power calculation. CDNR contributed to the conception and design of the study and wrote the funding application. SEM contributed to the development of the Goldilocks work principle, the conception and design of the study, and critical revision of the manuscript. LS contributed to the development of the Goldilocks work principle, the conception and design of the study, and critical revision of the manuscript. AH contributed to the development of the Goldilocks work principle, the conception and design of the study, wrote the initial protocol and the funding application, and is principle investigator of the trial. All authors read and commented on the draft version of the manuscript and approved the final version.

\section{Funding}

The study is peer reviewed and externally funded by the Danish Working Environment Research Fund (grant no. 18-2018-03). The funding source had no role in the design of the study and will not have any role in the implementation of the intervention, the data collection, data analyses, interpretation of the data, or decisions on when or where to report results.

\section{Availability of data and materials}

Not applicable.

\section{Ethics approval and consent to participate}

The Danish National Committee on Biomedical Research Ethics (The local ethical committee of Frederiksberg and Copenhagen) has evaluated a description of the study and concluded that, according to Danish law as defined in Committee Act $\S 2$ and $\S 1$, the intervention described should not be further reported to the local ethics committee (Ref number: $\mathrm{H}$ 18041423). Written informed consent will be collected for all individuals participating in the evaluation of the study.

\section{Consent for publication}

Not applicable.

\section{Competing interests}

The authors declare that they have no competing interests.

\section{Author details}

${ }^{1}$ National Research Centre for the Working Environment, Copenhagen, Denmark. ${ }^{2}$ Novo Nordisk A/S, Novo Nordisk Health \& Safety, Bagsværd, Denmark. ${ }^{3}$ Department of Public Health, Section of Social Medicine, University of Copenhagen, Copenhagen, Denmark. ${ }^{4}$ Department of Occupational Health Sciences and Psychology, Centre for Musculoskeletal Research, University of Gävle, Gävle, Sweden. ${ }^{5}$ School of Physiotherapy and Exercise Science, Curtin University, Perth, Western Australia, Australia. ${ }^{6}$ Institute of Sports Science and Clinical Biomechanics, University of Southern Denmark, Odense, Denmark.

Received: 17 January 2020 Accepted: 28 January 2020

Published online: 17 February 2020

\section{References}

1. Witten K. Geographies of obesity: environmental understandings of the obesity epidemic. 1st ed. Routledge; 2016. https://doi.org/10.4324/ 9781315584416

2. Holtermann A, Mortensen OS, Burr H, Søgaard K, Gyntelberg F, Suadicani P. Physical demands at work, physical fitness, and 30-year ischaemic heart disease and all-cause mortality in the Copenhagen male study. Scand J Work Environ Health. 2010;36:357-65.

3. Donkin A, Goldblatt P, Allen J, Nathanson V, Marmot M. Global action on the social determinants of health. BMJ Glob Health. 2018;3(Suppl 1):e000603.
4. Oakman J, Clays E, Jørgensen MB, Holtermann A. Are occupational physical activities tailored to the age of cleaners and manufacturing workers? Int Arch Occup Environ Health. 2019;92:185-93.

5. Straker L, Mathiassen SE. Increased physical work loads in modern work - a necessity for better health and performance? Ergonomics. 2009;52:1215-25.

6. Hallman D, Sato T, Kristiansen J, Gupta N, Skotte J, Holtermann A. Prolonged sitting is associated with attenuated heart rate variability during sleep in blue-collar workers. Int J Environ Res Public Health. 2015;12:14811-27.

7. Holtermann A, Jørgensen MB, Gram B, Christensen JR, Faber A, Overgaard K, et al. Worksite interventions for preventing physical deterioration among employees in job-groups with high physical work demands: background, design and conceptual model of FINALE. BMC Public Health. 2010;10:120.

8. Malik SH, Blake $H$, Suggs LS. A systematic review of workplace health promotion interventions for increasing physical activity. Br J Health Psychol. 2014;19:149-80.

9. Gay JL, Buchner DM, Smith J, He C. An examination of compensation effects in accelerometer-measured occupational and non-occupational physical activity. Prev Med Rep. 2017;8:55-9.

10. Jørgensen MB, Villadsen E, Burr H, Mortensen OS, Holtermann A. Does workplace health promotion in Denmark reach relevant target groups? Health Promot Int. 2015:30:318-27.

11. Morassaei S, Smith PM. Examining the relationship between psychosocial working conditions, physical work demands, and leisure time physical activity in Canada. J Occup Environ Med. 2011;53:1099-105.

12. Holtermann A, Mathiassen SE, Straker L. Promoting health and physical capacity during productive work: the goldilocks principle. Scand J Work Environ Health. 2019;45:90-7.

13. Straker L, Mathiassen SE, Holtermann A. The 'goldilocks principle': designing physical activity at work to be 'just right' for promoting health. Br J Sports Med. 2018:52:818-9.

14. Åstrand PO, Rodahl K, Dahl HA, Stromme SB. Physiological bases of exercise: textbook of work physiology. New York: McGraw-Hill Book Company; 1986.

15. Cumming T. Early childhood educators' well-being: an updated review of the literature. Early Childhood Educ J. 2017:45:583-93.

16. Linnan L, Arandia G, Bateman LA, Vaughn A, Smith N, Ward D. The health and working conditions of women employed in child care. Int J Environ Res Public Health. 2017;14. Article number 283.

17. Otten JJ, Bradford VA, Stover B, Hill HD, Osborne C, Getts K, et al. The culture of health in early care and education: workers' wages, health, and job characteristics. Health Aff (Millwood). 2019;38:709-20.

18. Ward DS, Vaughn AE, Hales D, Viera AJ, Gizlice Z, Bateman LA, et al. Workplace health and safety intervention for child care staff: rationale, design, and baseline results from the CARE cluster randomized control trial. Contemp Clin Trials. 2018:68:116-26.

19. Physical Activity Guidelines Advisory Committee. Physical activity guidelines advisory committee scientific report. Wash DC US Dep Health Hum Serv. 2018;2018:F2-33.

20. Bonis M, Loftin M, Ward D, Tseng TS, Clesi A, Sothern M. Improving physical activity in daycare interventions. Child Obes. 2014;10:334-41.

21. Chang-Martinez C, Ahmed NU, Natale RA, Messiah SE. State-mandated nutrition, physical activity, and screen time policies in child care centers. Health Promot Pract. 2018;19:411-7.

22. Chan A-W, Tetzlaff JM, Altman DG, Laupacis A, Gøtzsche PC, Krleža-Jerić K, et al. SPIRIT 2013 statement: defining standard protocol items for clinical trials. Ann Intern Med. 2013:158:200.

23. Hoffmann TC, Glasziou PP, Boutron I, Milne R, Perera R, Moher D, et al. Better reporting of interventions: template for intervention description and replication (TIDieR) checklist and guide. BMJ. 2014;348:g1687.

24. Moher D, Hopewell S, Schulz KF, Montori V, Gøtzsche PC, Devereaux PJ, et al. CONSORT 2010 explanation and elaboration: updated guidelines for reporting parallel group randomised trials. J Clin Epidemiol. 2010;63:e1-37.

25. Schelvis RM, Oude Hengel KM, Burdorf A, Blatter BM, Strijk JE, van der Beek AJ. Evaluation of occupational health interventions using a randomized controlled trial: challenges and alternative research designs. Scand J Work Environ Health. 2015;41:491-503.

26. Mdege ND, Man M-S, Taylor Nee Brown CA, Torgerson DJ. Systematic review of stepped wedge cluster randomized trials shows that design is particularly used to evaluate interventions during routine implementation. J Clin Epidemiol. 2011;64:936-48.

27. R Foundation for Statistical Computing. The R Project for Statistical Computing. https://www.R-project.org/. Accessed 3 Jan 2020. 
28. Kuorinka I, Jonsson B, Kilbom A, Vinterberg H, Biering-Sørensen F, Andersson G, et al. Standardised Nordic questionnaires for the analysis of musculoskeletal symptoms. Appl Ergon. 1987;18:233-7.

29. Ware JE, Snow KK, Kosinski M. SF-36 version 2 health survey: manual and interpretation guide. Linc RI Qual Metr Inc. 2000.

30. Ware JE Jr. SF-36 health survey update. Spine. 2000:25:3130-9.

31. Schwarzer R, Bäßler J, Kwiatek P, Schröder K, Zhang JX. The assessment of optimistic self-beliefs: comparison of the German, Spanish, and Chinese versions of the general self-efficacy scale. Appl Psychol. 1997;46:69-88.

32. Topp CW, Østergaard SD, Søndergaard S, Bech P. The WHO-5 well-being index: a systematic review of the literature. Psychother Psychosom. 2015;84:167-76.

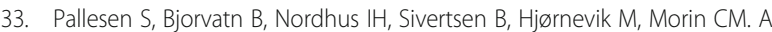
new scale for measuring insomnia: the Bergen insomnia scale. Percept Mot Skills. 2008:107:691-706.

34. Strøyer J, Jensen LD, Avlund K, Essendrop M, Warming S, Schibye B. Validity and reliability of self-assessed physical fitness using visual analogue scales. Percept Mot Skills. 2007;104:519-33.

35. Cohen S, Kamarck T, Mermelstein R. A global measure of perceived stress. J Health Soc Behav. 1983;24:385.

36. Borg G. Perceived exertion as an indicator of somatic stress. Scand J Rehabil Med. 1970;2:92-8.

37. Kessler RC, Barber C, Beck A, Berglund P, Cleary PD, McKenas D, et al. The World Health Organization Health and Work Performance Questionnaire (HPQ). J Occup Environ Med. 2003;45:156-74.

38. Clausen T, Madsen IE, Christensen KB, Bjorner JB, Poulsen OM, Maltesen T, et al. The Danish psychosocial work environment questionnaire (DPQ): development, content, reliability and validity. Scand J Work Environ Health. 2019:45:356-69

39. Tuomi K, Ilmarinen J, Jahkola A, Katajarinne L, Tulkki A. Work ability index. Finnish Institute of Occupational Health: Helsinki; 1998.

40. Stevens ML, Crowley P, Garde AH, Mortensen OS, Nygård C-H, Holtermann A. Validation of a short-form version of the Danish need for recovery scale against the full scale. Int J Environ Res Public Health. 2019;16:2334.

41. Aboagye E, Jensen I, Bergström G, Hagberg J, Axén I, Lohela-Karlsson M. Validity and test-retest reliability of an at-work production loss instrument. Occup Med. 2016;66:377-82.

42. Lohela Karlsson M, Bergström G, Björklund C, Hagberg J, Jensen I. Measuring production loss due to health and work environment problems: construct validity and implications. J Occup Environ Med. 2013;55:1475-83.

43. Ingebrigtsen J, Stemland I, Christiansen C, Skotte J, Hanisch C, Krustrup P. Validation of a commercial and custom made accelerometer-based software for step count and frequency during walking and running. J Ergon. 2013;3. Article number 119

44. Skotte J, Korshøj M, Kristiansen J, Hanisch C, Holtermann A. Detection of physical activity types using Triaxial accelerometers. J Phys Act Health. 2014; 11:76-84.

45. Stemland I, Ingebrigtsen J, Christiansen CS, Jensen BR, Hanisch C, Skotte J, et al. Validity of the Acti4 method for detection of physical activity types in free-living settings: comparison with video analysis. Ergonomics. 2015;58: 953-65.

46. Parak J, Korhonen I. Accuracy of Firstbeat bodyguard 2 beat-to-beat heart rate monitor. White Pap Firstbeat Technol Ltd. 2013.

47. Ekblom-Bak E, Björkman F, Hellenius M-L, Ekblom B. A new submaximal cycle ergometer test for prediction of $\mathrm{VO}_{2 \text { max }}$ : a new submaximal cycle ergometer test. Scand J Med Sci Sports. 2014;24:319-26.

48. Kigozi J, Jowett S, Lewis M, Barton P, Coast J. Estimating productivity costs using the friction cost approach in practice: a systematic review. Eur J Health Econ. 2016;17:31-44.

49. Ferm L, Rasmussen CDN, Jørgensen MB. Operationalizing a model to quantify implementation of a multi-component intervention in a steppedwedge trial. Implement Sci. 2018;13:26.

50. Steckler $A B$, Linnan $L$. Process evaluation for public health interventions and research. San Francisco, CA: Jossey-Bass; 2002.

51. Rasmussen CDN, Hendriksen PR, Svendsen MJ, Ekner D, Hansen K, Sørensen $\mathrm{OH}$, et al. Improving work for the body - a participatory ergonomic intervention aiming at reducing physical exertion and musculoskeletal pain among childcare workers (the TOY-project): study protocol for a wait-list cluster-randomized controlled trial. Trials. 2018;19:411.
52. Pedišić Ž. Measurement issues and poor adjustments for physical activity and sleep undermine sedentary behaviour research — the focus should shift to the balance between sleep, sedentary behaviour, standing and activity. Kinesiol Int J Fundam Appl Kinesiol. 2014:46:135-46.

53. Pedišić Ž, Dumuid D. S olds T. integrating sleep, sedentary behaviour, and physical activity research in the emerging field of time-use epidemiology: definitions, concepts, statistical methods, theoretical framework, and future directions. Kinesiol Int J Fundam Appl Kinesiol. 2017;49:252-69.

54. Gupta N, Mathiassen SE, Mateu-Figueras G, Heiden M, Hallman DM, Jørgensen MB, et al. A comparison of standard and compositional data analysis in studies addressing group differences in sedentary behavior and physical activity. Int J Behav Nutr Phys Act. 2018;15. https://doi.org/10.1186/ s12966-018-0685-1.

55. Hron K, Filzmoser $\mathrm{P}$, Thompson K. Linear regression with compositional explanatory variables. J Appl Stat. 2012:39:1115-28.

56. Lund Rasmussen C, Palarea-Albaladejo J, Korshøj M, Gupta N, Nabe-Nielsen K, Holtermann A, et al. Is high aerobic workload at work associated with leisure time physical activity and sedentary behaviour among blue-collar workers? A compositional data analysis based on accelerometer data. PLoS One. 2019:14:e0217024.

57. Twisk JW. Applied multilevel analysis: a practical guide for medical researchers: Cambridge university press; 2006.

58. Speklé EM, Heinrich J, Hoozemans MJ, Blatter BM, van der Beek AJ, van Dieën $J \mathrm{H}$, et al. The cost-effectiveness of the RSI QuickScan intervention programme for computer workers: results of an economic evaluation alongside a randomised controlled trial. BMC Musculoskelet Disord. 2010;11. https://doi.org/10.1186/1471-2474-11-259.

59. Lachin JM. Statistical considerations in the intent-to-treat principle. Control Clin Trials. 2000;21:167-89.

60. Hallman DM, Mathiassen SE, van der Beek AJ, Jackson JA, Coenen P Calibration of self-reported time spent sitting, standing and walking among office workers: a compositional data analysis. Int J Environ Res Public Health 2019:16:3111.

61. da Silva DRP, Ohara D, da Silva-Grigoletto ME. The importance of an integrative view of behaviors adopted during the 24 hours of the day in physical activity and exercise interventions studies. Rev Bras Cineantropometria Desempenho Hum. 2019;21.

62. Landsberger $\mathrm{H}$. Hawthorne revisited: management and the worker, its critics, and developments in human relations in industry. Ithaca, NY: Cornell University; 1958

\section{Publisher's Note}

Springer Nature remains neutral with regard to jurisdictional claims in published maps and institutional affiliations.

Ready to submit your research? Choose BMC and benefit from:

- fast, convenient online submission

- thorough peer review by experienced researchers in your field

- rapid publication on acceptance

- support for research data, including large and complex data types

- gold Open Access which fosters wider collaboration and increased citations

- maximum visibility for your research: over $100 \mathrm{M}$ website views per year

At BMC, research is always in progress.

Learn more biomedcentral.com/submissions 